\title{
Fabrication of noble-metal nanoconstrictions and observation of conductance fluctuations
}

\author{
E. Scheer and H. v. Löhneysen \\ Physikalisches Institut, Universität Karlsruhe, Engesserstrasse 7, 76128 Karlsruhe, Germany \\ H. Hein \\ Kernforschungszentrum Karlsruhe, Institut für Mikrostrukturtechnik, Postfach 3640, 76021 Karlsruhe. \\ Germany
}

(Received 7 March 1994; accepted 17 September 1994)

We describe the fabrication of planar metallic nanostructures with lateral dimensions down to about $80 \mathrm{~nm}$ and thicknesses varying from 15 to $50 \mathrm{~nm}$. We use a lift-off process with a polymethylmethacrylate bilayer resist on silicon substrate and an electron beam writer system for microfabrication. The noble-metal layers are deposited by thermal evaporation. The geometries are point contacts, rings, and wires in two-lead configuration. We discuss measurements of the magnetoconductance at very low temperatures and in magnetic fields up to $4 \mathrm{~T}$ in dependence of the width of the samples. Here the electron transport is in the diffusive regime and the width dependence of the universal conductance fluctuations is investigated.

\section{INTRODUCTION}

Metallic systems with reduced geometry show new electronic phenomena evoked by quantum interference, e.g. fluctuations of the magnetoconductance of the order of a universal value $e^{2} / h$ or oscillations of the magnetoconductance of ring structures.' These properties can be observed if the electron waves are able to interfere. This means that the dimensions of the sample must be of the order of magnitude of the phase coherence length $l_{\varphi}$ or smaller.

For metals, this condition is fulfilled in small structures of lateral dimensions $L<1 \mu \mathrm{m}$ at very low temperatures $T<1$ K. Because usually the elastic mean free path $l_{e}(10$ $\left.\mathrm{nm}<l_{e}<50 \mathrm{~nm}\right)$ is still smaller than the sample size $L$, the electronic transport is in the diffusive regime where the interference of diffusely scattered electron waves leads to universal conductance fluctuations (UCF), i.e., reproducible but stochastic variations of the electrical conductance as a function of the magnetic field or the electrochemical potential. ${ }^{2}$

The theoretically expected root-mean-square (rms) amplitude $\Delta G$ of the UCFs is about $e^{2} / h$ and depends slightly on the dimensionality. ${ }^{2}$ For one dimension $\Delta G_{1 \mathrm{D}}=0.729 e^{2} / h$, for two dimensions $\Delta G_{2 \mathrm{D}}=0.862 e^{2} / \mathrm{h}$, and for three dimensions $\Delta G_{3 \mathrm{D}}=1.088 e^{2} / h$ are calculated. These amplitudes are reduced in magnetic fields by a factor $2^{1 / 2}$ due to the suppression of the Cooperon channel. With the same assumptions as in weak (anti-) localization theory one obtains a further factor-of- 2 reduction in amplitude due to the spinorbit scattering in metals with large atomic number. Thus $\Delta G_{1 \mathrm{D}}=0.26 e^{2} / h$ would be expected for gold wires, and $\Delta G_{2 \mathrm{D}}=0.30 e^{2} / h$ for small two-dimensional gold films.

Experiments on long (one-dimensional) gold and silver samples ${ }^{3,4}$ show a rms amplitude of $0.12 e^{2 / h}$ for two-lead measurements with large contact areas. This is a factor of 2 smaller than the expectation. The reduction is explained by the authors in terms of a reduced backscattering probability of electrons diffusing into the contact leads. For this contact geometry the fluctuations should be symmetric in the field. ${ }^{5}$ The characteristic fluctuation "period" $B_{c}$, the so-called cor- relation field, is a measure for the area $A$ surrounded by typical interfering electronic paths since $A B_{c}=\Phi_{0}$ with $\Phi_{0}=h / e,^{2}$ the elementary flux quantum. Because all paths shorter than $l_{\varphi}$ contribute to the interference pattern, ${ }^{6} B_{c}$ should depend on the geometry of the samples if the width and/or the length of the samples become smaller than $l_{\varphi}$. In addition, the energy range $e V_{m}\left(V_{m}\right.$ is the measuring voltage) around the Fermi energy where the electrons contributing to the transport come from, generates a new energy scale. This energy characterizes the Thouless length $L_{T}=\left(h D / e V_{m}\right)^{1 / 2}$. If $L_{T}$ becomes shorter than $l_{\varphi}, L_{T}$ limits the observability for interference of the electrons and determines the magnitude of the fluctuations.

The dependence of the characteristic features of the UCFs, $B_{c}$ and rms amplitude, on the geometry are not yet understood completely. In particular, the influence of the contact geometry on the amplitude and the dependence of $B_{c}$ on the width of the sample have not been investigated systematically up to now.

We will report on measurements of the magnetoconductance, so-called "magnetofingerprints," of gold samples in two-lead configuration with short and wide leads and typical sample dimensions between $80 \mathrm{~nm}$ and $5 \mu \mathrm{m}$ where the geometry of both samples and leads determines $B_{c}$.

\section{SAMPLE FABRICATION}

The present route of preparation is aimed at producing the nanostructures and the macroscopic contact pads simultaneously within one single step for exposure, development, metallization and lift-off in order to avoid overlay and interface problems.

Undoped 4-in.-diam silicon wafers are used as substrates. The wafers are exposed to $100 \%$ hexamethyldisilazane (HMDS) vapor at $130^{\circ} \mathrm{C}$ in a vacuum oven to improve the adhesion to the resist system. The resist system consists of a double layer of polymethylmethacrylate (PMMA), where as first layer PMMA with a molecular mass of $600 \mathrm{~kg} / \mathrm{mol}$ and a solids content of $3 \%$ dissolved in chlorobenzene is spin 
coated to a thickness of $200 \mathrm{~nm}$. In order to remove residual solvent a prebake for $30 \mathrm{~min}$ at $170^{\circ} \mathrm{C}$ is performed. A higher molecular mass of $950 \mathrm{~kg} / \mathrm{mol}$ with a lower solids content of $2 \%$ is used for the toplayer. With a dynamic dis pense of the toplayer PMMA no thinning out of the first layer is observed. After a second prebake step with the same parameters a total resist thickness of about $300 \mathrm{~nm}$ is measured.

For the exposure a commercial electron beam lithography machine EBPG5-HS (Leica-Cambridge) is used. It is a highspeed vector scan machine with a maximum acceleration voltage of $100 \mathrm{kV}$, which results in a low percentage of backscattered electrons and supports the exposure of patterns with lateral dimensions $L<1 \mu \mathrm{m}$. The e-beam operates with a $\mathrm{LaB}_{6}$ flat tip cathode with an original $20 \mu \mathrm{m}$ diameter truncation. This improves the cathode lifetime but limits the brightness: consequently very small spot diameter (30-50 nmi and beam current $<1-4 \mathrm{nA}$ are difficult to establish. Both the coarse contact leads and pads of about $1 \mathrm{~mm}^{2}$ size and the nanostructures with dimensions down to $80 \mathrm{~nm}$ are exposed in the same run but with different e-beam parameters. For the coarse structures, a beam step size of $250 \mathrm{~nm}$ with a current of about $200 \mathrm{nA}$ and a spot size of $300 \mathrm{~nm}$ diam is used. The exposure dose for these structures is 700 $\mu \mathrm{C} / \mathrm{cm}^{2}$. For the fine structures the parameters are: beam step size $20 \mathrm{~nm}$; current $2 \mathrm{nA}$; nominal spot size $40 \mathrm{~nm}$; and exposure dose $1300 \mu \mathrm{C} / \mathrm{cm}^{2}$. After the exposure the PMMA is developed in a bath of $75 \%$ isopropanol (IPA) and $25 \%$ isobutylmethyl ketone (MIBK) at $30^{\circ} \mathrm{C}$ for about $5 \mathrm{~s}$ and rinsed for around $1 \mathrm{~min}$ in pure IPA. The bilayer resist system together with the slightly enhanced developing temperature cause an undercut profile of the resist edges suitable for the lift-oft following later in the manufacturing process.

Because the adhesion of gold and silver on the silicon wafer covered by its native oxide layer is rather poor, a primary treatment of the developed substrate is necessary before depositing the metal film. Therefore the substrate is etched in a solution of $80 \%$ de-ionized (di) water with $25 \%$ HF at room temperature for $15 \mathrm{~s}$, rinsed in di-water for $30 \mathrm{~s}$, and then coated with $n$-decane alcohol in order to avoid reoxidization of the cleaned surface. Then the substrate is immediately mounted in the lock chamber of an ultrahigh vacuum (UHV) system with exposure to air for less than 5 min. The alcohol evaporates during pumping down of the system in about $15 \mathrm{~min}$ without residue. At a pressure of $10^{-\mathrm{K}} \mathrm{hPa}$ a $1.3 \mathrm{-nm}$ thin germanium seeded layer is e-gun evaporated. Then the substrate is cooled down to about $-20{ }^{\circ} \mathrm{C}$ and the noble-metal layer with a thickness of $15-50$ $\mathrm{nm}$ is thermally evaporated at rates of $0.01-0.02 \mathrm{~nm} / \mathrm{s}$. The evaporation at reduced substrate temperatures leads to a slightly worse adhesion of the metal film to the substrate but together with the low evaporation rate a much better quality of the tilm is achieved, i.e., smaller grain size, mechanical stability, and smoothness, which is favorable for the lift-off process. Finally, the lift-off is performed in acetone at room temperature for $2 \mathrm{~h}$. Short ultrasonic treatment for a few seconds improves the sample quality.
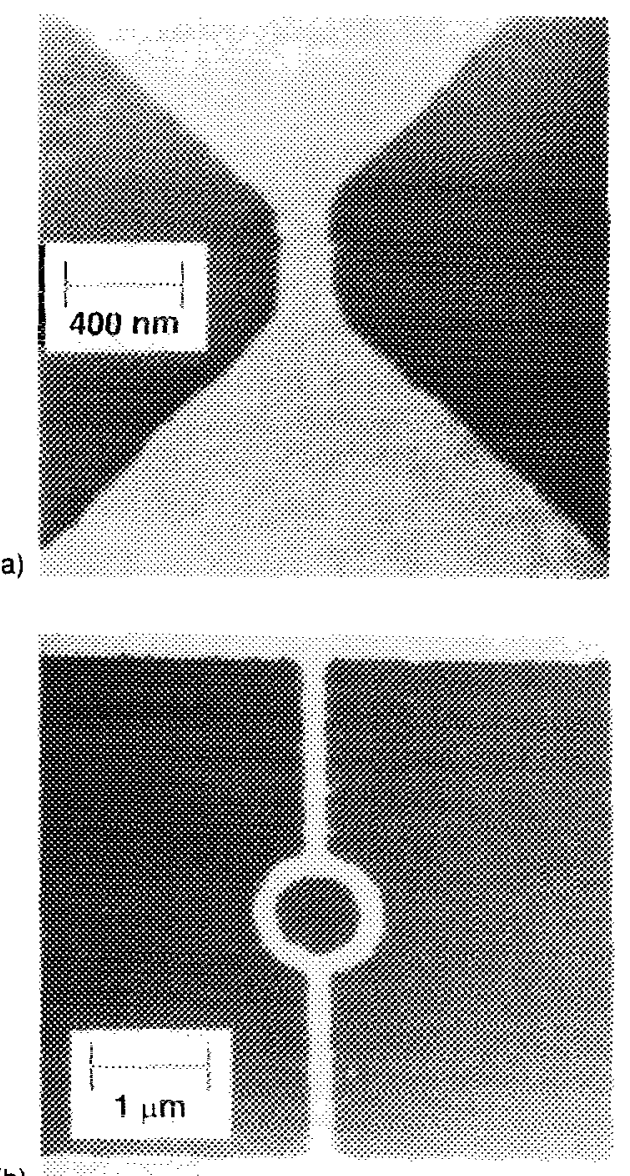

(b)

Fici. 1. SEM picture of (a) sample D1-2. (b) sample D+-2. Magnification as indicated by the bars.

\section{MEASUREMENTS}

After the preparation, the structures are inspected in a scanning electron microscope (SEM) with a magnification of 50000 , the metal layer thickness is checked by mechanical step height measurement, and the wafer is cut into chips of $12 \mathrm{~mm} \times 12 \mathrm{~mm}$ size each carrying an array of $4 \times 4$ samples with the same contact geometry but different samples on a mesoscopic scale (Fig. 1). Electrical contacts to the sample are accomplished by spring contact pins which are pressed onto the pads. The mesoscopic geometries of the samples are as follows: point contacts with width $w$ from $80 \mathrm{~nm}$ up to $560 \mathrm{~nm}$ and wires with width from 80 to $340 \mathrm{~nm}$ and length $L$ from 100 to $400 \mathrm{~nm}$ are investigated. In addition a ring structure with line width $w=150 \mathrm{~nm}$, inner diameter $750 \mathrm{~nm}$, outer diameter $1050 \mathrm{~nm}$ and a long wire with length $L=91$ $\mu \mathrm{m}$, width $w=220 \mathrm{~nm}$ are designed. The typical classical resistance of the samples at low temperatures is between 15 and $100 \Omega$ for the short wires, point contacts, and rings, and around $1300 \Omega$ for the long wire. From the residual resistiv ity ratio $\mathrm{RRR}=R(T=300 \mathrm{~K}) / R(T \rightarrow 0) \approx 1+l_{\mathrm{ph}} / l$, and the phonon scattering length of gold at room temperature ${ }^{7}$ we deduce elastic mean free paths from 23 to $27 \mathrm{~nm}$. With this value we calculate the electronic diffusion constant $D=v_{F} l / 3 \approx 0.011 \mathrm{~m}^{2} / \mathrm{s}$.

The measurements of the magnetoconductance are per- 


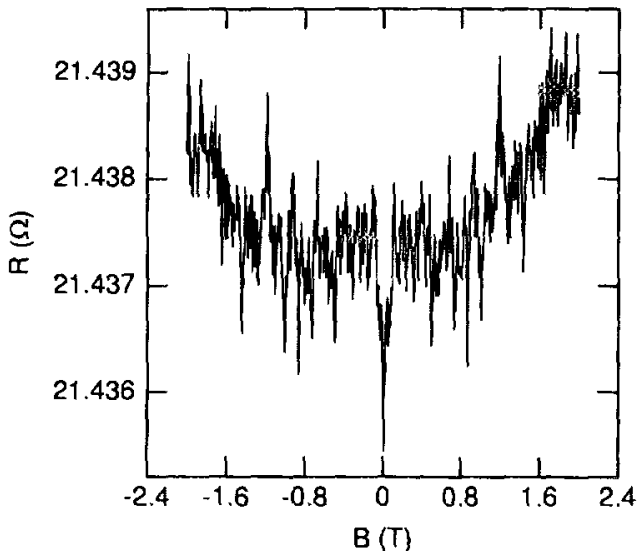

Fig. 2. Magnetoresistance (magnetofingerprint) of sample D3 for $-2 \mathrm{~T} \leqslant B \leqslant 2 \mathrm{~T}$.

formed with the sample mounted in the mixing chamber of a ${ }^{3} \mathrm{He} /{ }^{4} \mathrm{He}$ dilution refrigerator. In order to avoid eddy-current heating when sweeping the field the mixing chamber is made of araldite. The magnetoresistance is measured with an acresistance bridge with excitation currents of about $3 \mu \mathrm{A}$. Typical voltage changes corresponding to the UCF signals are some nanovolts. Lowest measuring temperatures are approximately $30 \mathrm{mK}$

\section{RESULTS AND DISCUSSION}

Figure 2 shows the magnetoresistance of the gold sample D3 with $L=400 \mathrm{~nm}, w=340 \mathrm{~nm}$ and $d=18 \mathrm{~nm}$ for magnetic fields $-2 \mathrm{~T} \leqslant B \leqslant 2 \mathrm{~T}$ (Dimensions, measurement parameters, and results for all investigated samples are listed in Table I) The absolute resistance at $T=30 \mathrm{mK}$ is $21.44 \Omega$. Because the samples are prepared in the one-step process described above, the leads and the samples themselves have the same thickness and therefore the same sheet resistance $R_{\square}=3.1 \Omega$ This results in an offset resistance $R_{o}$ in series with the sample resistance $R_{\mathrm{s}}$. From the geometry of sample and leads and the measured resistances we can estimate the size of $R_{0}$ as $16.1 \Omega$. It is not clear to what amount $R_{0}$ should contribute to the UCFs. Therefore, in the following discussion of the rms amplitudes we assume that $R_{o}$ does not fluctuate at all. We scale the measured resistance fluctuations $\Delta R$ by the sample resistance $R_{S}=R-R_{0}$ to obtain the conductance fluctuations $\Delta G=-\Delta R / R_{s}^{2}$.

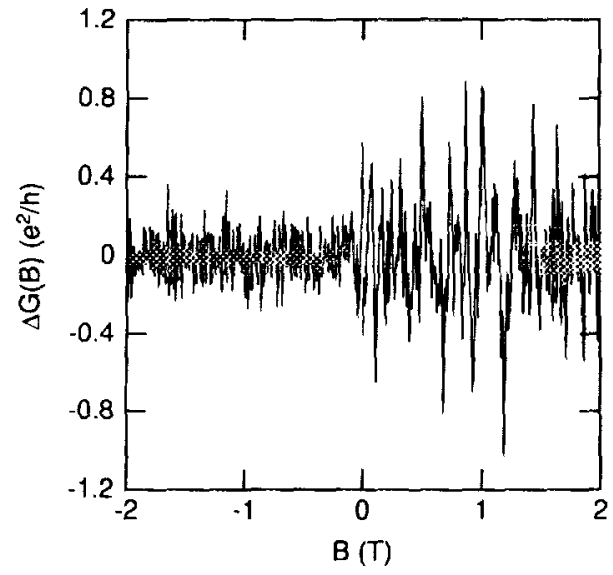

FIG. 3. Symmetric part $[\Delta G(B)+\Delta G(-B)] / 2$ (right-hand trace) and antisymmetric part $[\Delta G(B)-\Delta G(-B)] / 2$ (left-hand trace) of the conductance fluctuations of Fig. 2. The resistance fluctuations have been scaled to obtain conductance fluctuations by $\Delta G=-\Delta R / R_{s}^{2}$ with $R_{s}=5.3 \Omega$

Around $B=0$ one observes a sharp minimum due to weak antilocalization. A fit to the $2 \mathrm{D}$ theory yields $l_{e}=19 \mathrm{~nm}$ in rather good agreement with the value deduced from the RRR. In addition to this anomaly which is subtracted in the following analysis one observes fluctuations of the conductance which are symmetric in the field, as theory predicts. We use the symmetry in order to estimate the experimental noise. The left trace in the Fig. 3 shows the antisymmetric part $[\Delta G(B)-\Delta G(-B)] / 2$ which should represent the experimental noise and the right trace includes the symmetric part $[\Delta G(B)+\Delta G(-B)] / 2$, i.e., the UCF signal and the symmetric part of the noise. Figure 4 shows the autocorrelation functions of the antisymmetric part, $C_{A A}(\Delta B)$ together with the autocorrelation functions of the magnetofingerprints for negative field and positive field, $C_{n n}(\Delta B)$ and $C_{p p}(\Delta B)$, and the cross-correlation function of the traces with negative and positive field $C_{n p}(\Delta B)$. The symmetric and antisymmetric parts of the noise should have the same size, which is indeed roughly the case as checked by a detailed analysis. We obtain the rms amplitude of the experimental noise by $\left[C_{A A}(0)\right]^{1 / 2} \approx 1 \mathrm{nV}$. The value $C_{n p}(\Delta B=0)$ gives the rms amplitude of the fluctuations $\Delta G=\left[C_{n p}(0)\right]^{1 / 2}=0.27 e^{2} / h$, which is a little smaller than the theoretical value $\Delta G_{2 \mathrm{D}}=0.3$ $e^{2} / h$ and larger by a factor 2.3 than the observations ${ }^{3,4}$ for samples with wide leads. The half-width of the maximum of

TABLE I. Dimensions, measuring parameters, and results for gold samples C4, DI-2, D2-2, D3, D4, and D4-2. The meanings of the symbols are: $L$ : sample length, $w$ : width, $d$ : thickness, $R:$ measured resistance, $R_{o}:$ offset resistance as discussed in the text. $R_{s}:$ sample resistance $R-R_{o}, T:$ measuring temperature, $I_{m}$ : measuring current, $V_{m}$ : measuring voltage at $R_{s}, \Delta G ;$ rms amplitude of the UCF, $B_{r}:$ correlation field of the UCF, $K$ : relative correlation between magnetofingerprint for positive and negative applied magnetic fields.

\begin{tabular}{|c|c|c|c|c|c|c|c|c|c|c|c|c|}
\hline Sample & $L(\mathrm{~nm})$ & $w(\mathrm{~nm})$ & $d(\mathrm{~nm})$ & $R(\Omega)$ & $R_{o}(\Omega)$ & $R_{\mathrm{s}}(\Omega)$ & $T(\mathrm{mK})$ & $I_{m}(\mu \mathrm{A})$ & $V_{m}(\mu V)$ & $\Delta G\left(e^{2} / h\right)$ & $B_{c}(\mathrm{mT})$ & $K(\%)$ \\
\hline $\mathrm{C} 4$ & 91000 & 220 & 18 & 1307 & 8 & 1299 & 30 & 0.1 & 130 & 0.0002 & $\cdots$ & 86 \\
\hline Dl-2 & 400 & 160 & 18 & 24.5 & 14.4 & 10.1 & 140 & 3 & 30 & 0.19 & 27 & 78 \\
\hline $\mathrm{D} 2-2$ & 400 & 240 & 18 & 22.4 & 14.4 & 8.0 & 25 & 3 & 24 & 0.24 & 18 & 75 \\
\hline D3 & 400 & 340 & 18 & 21.4 & 16.1 & 5.3 & 30 & 4 & 21 & 0.27 & 12 & 92 \\
\hline D4 & 4700 & 150 (ring) & 18 & 91.5 & 8.0 & 83.5 & 30 & 3 & 250 & 0.01 & 25 & 92 \\
\hline D4-2 & 4700 & 150 (ring) & 18 & 83.0 & 7.5 & 75.5 & 30 & 3 & 227 & 0.01 & 27 & 91 \\
\hline
\end{tabular}




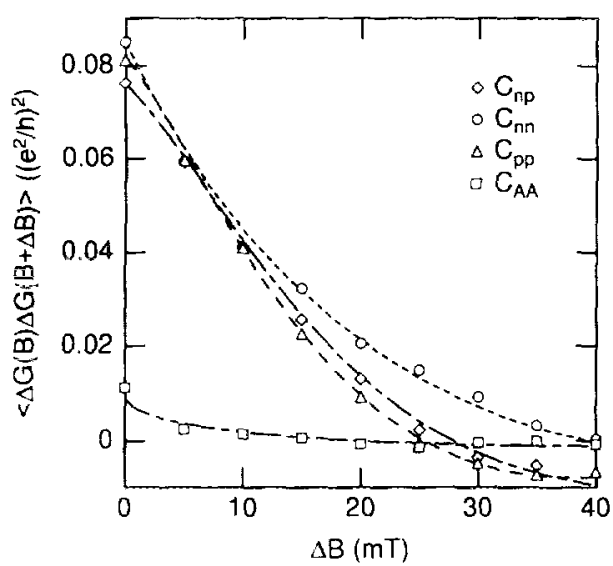

Flg. 4. Correlation functions $C_{n p}, C_{n n}, C_{p n}$, and $C_{A A}$, of the conductance fluctuations of Fig. 3. The half-width of $C_{n P}(\Delta B)$ indicates the correlation field $B_{1}=12 \mathrm{mT}$. Symbols indicate calculated correlation functions (in in tervals of $5 \mathrm{mT}$. Lines are guide to the eye.

$C_{n p}(\Delta B)$ gives the correlation field $B_{c}$. In this sample we obtain $B=12 \mathrm{mT}$. The last column of Table I contains the correlation $K=C_{n p}(0) /\left[C_{n \prime \prime}(0) C_{n \prime \prime}(0)\right]^{1 / 2}$, which is a measure for the reproducibility of the fingerprints and is in our examples always better than $75 \%$. The reproducibility is also seen in the overall agreement $C_{n n}(\Delta B), C_{p p}(\Delta B)$, and $C_{n p}(\Delta B)$ in Fig. 4 .

For samples much shorter and narrower than the minimum of $L_{T}$ and $l_{\varphi}, B_{c}$. should be nearly independent of the length and the width because the interference patterns are characterized by electrons diffusing into the contact pads. For samples with $w<l_{\varphi}$ and $L \geqslant \min \left(l_{\varphi}, L_{T}\right), B_{c}$ should be inversely proportional to $w: B_{C}=h /\left[e w \min \left(l_{\varphi}, L_{T}\right)\right]$. This is observed for samples D1-2, D2-2, and D3 with $w=160$, 240, and $340 \mathrm{~nm}, B_{c}=27,18$, and $12 \mathrm{mT}$, respectively, although $L=400 \mathrm{~nm}$ is smaller than $\min \left(l_{\varphi}, L_{T}\right)$. With these values we obtain $\min \left(l_{4}, L_{T}\right) \approx 1 \mu \mathrm{m}$. For sample $\mathrm{D} 3$ the measuring voltage was $21 \mu \mathrm{V}$. Thus we deduce $L_{T} \approx 1.4 \mu \mathrm{m}$ in consistence with the value obtained from the fluctuations.

For the ring sample we also observe aperiodic conductance fluctuations (Fig. 5) with amplitude $\Delta G=0.01 e^{2} / \mathrm{h}$ and $B_{c}=25 \mathrm{mT} . B_{c}$ is in agreement with the result for sample D1-2 with nearly the same width. Because of the relatively high measuring voltage $V_{m}=250 \mu \mathrm{V}$, the Thouless length $L_{T}=\left(h D / e V_{m}\right)^{1 / 2} \approx 430 \mathrm{~nm}$ in this case is much smaller. For narrow samples $\left(w \ll L_{T}\right)$ longer than $L_{T}$ the magnetofingerprint should be an incoherent superposition of $N=L / L_{T}$ independent samples with same width and thus same $B_{c}$. Therefore the amplitude of the UCF should be reduced by $\Delta G / \Delta G_{0}=\left(L_{T} / L\right)^{3 / 2} \approx 0.03$ (Ref. 6) for this sample with $L=L_{\text {eff }} \approx 4.7 \mu \mathrm{m}$ (leads about $1.7 \mu \mathrm{m}$ each, half-perimeter about $1.3 \mu \mathrm{m}$ ). This yields an unaveraged $\mathrm{rms}$ amplitude $\Delta G_{0}=0.33 e^{2} / h$ which is again somewhat higher than the theoretical value.

We do not observe periodic Aharonov-Bohm oscillations for the ring sample expected for multiples of $B=6 \mathrm{mT}$, presumably because of the following two reasons: (1) the aspect ratio between the large linewidth $w$ of $150 \mathrm{~nm}$ and the outer

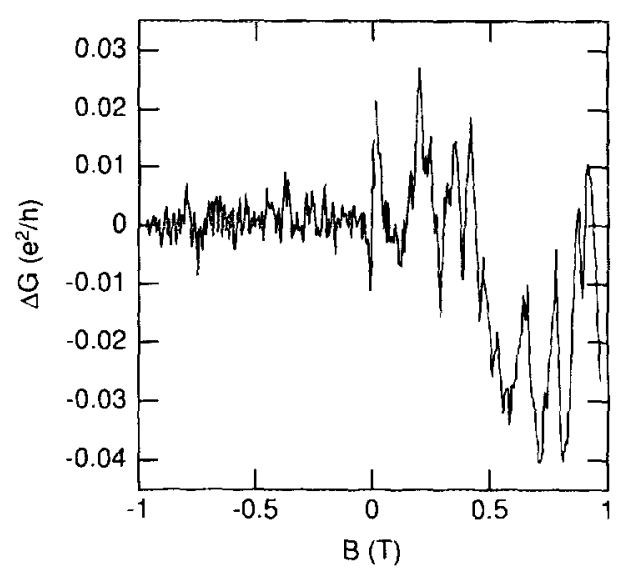

FIG. 5. Symmetric part $[\Delta G(B)+\Delta G(-B)] / 2$ (right-hand trace) and antisymmetric part $[\Delta G(B)-\Delta G(-B)] / 2$ (left-hand trace) of the conductance fluctuations of sample D4

ring diameter $d$ is unfavorable. $w$ should be less than 50 $\mathrm{nm} ;{ }^{7}(2)$ the amplitude of the oscillations is damped with $\exp \left(-\pi d / L_{T}\right)$ because interference only occurs for electrons which surround one ring branch without loosing their phase coherence.

Another interesting observation is made in the magnetoconductance of sample D4. Figure 6 shows two magnetofingerprints of sample D4-2 (same geometry as D4, but different $R=81.5 \Omega$ ). The traces have been recorded on two different days without warming up the sample, but switching short cuts open and closed in the leads. The cross correlations of the positive and negative parts of each trace are better than $90 \%$, but the patterns have changed slightly from the first measurement (upper curve) to the second measurement (lower curve). For low fields $|B|<1.7 \mathrm{~T}$ the pattern is nearly unchanged, for higher fields characteristic deviations occur, e.g., at $|B| \approx 1.85 \mathrm{~T}$. This means that the changing of some single scattering centers does not change the whole fluctuation pattern, presumably because the number of trans-

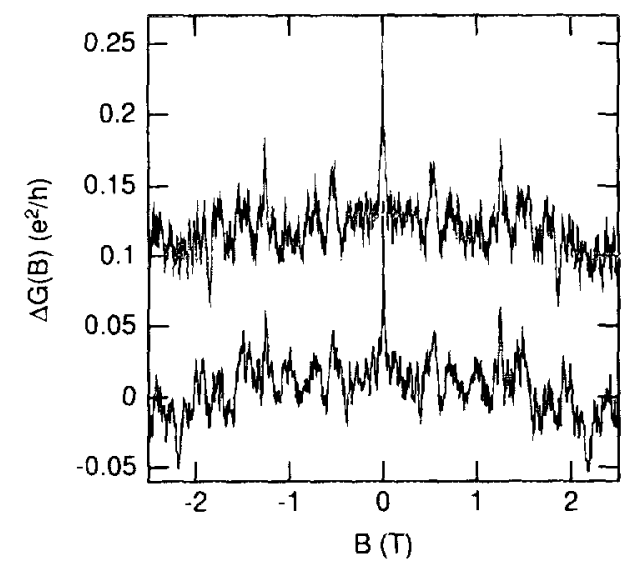

Fic. 6. Comparison of two magnetofingerprints of sample D4-2 with same measurement parameters. Traces are offset for clarity. 
port channels in our sample is still very large, so that most of the electron scattering paths remain unaffected.

For completeness we mention the result for the long wire C4 with $L=91 \mu \mathrm{m}$ : We observe fluctuations of the magnetoconductance with amplitude $\Delta G=0.0002 e^{2} / h$ in qualitative agreement with the expected reduction by a factor $\approx 1900$ for $L>I_{4}$ and $L>L_{T} \approx 600 \mathrm{~nm}$. A reliable result for $B_{c}$ cannot be obtained because the scatter of the data is too large for the small amplitude of $\Delta G$.

\section{CONCLUSIONS}

We have presented a process to fabricate noble-metal nanostructures with lateral dimensions down to $80 \mathrm{~nm}$ and contact lead dimensions up to $1 \mathrm{~mm}$ simultaneously. A bilayer resist system was exposed with $100 \mathrm{kV}$ acceleration voltage. For metallization thermal evaporation of noble metal followed by lift-off is used.

Our preliminary investigations of quantum interference effects in these gold samples are reported. Conductance fluctuations have been observed for all investigated samples with dimensions up to several micrometers. From the absolute resistances and the residual resistance ratio $R R R$ we deduce an offset resistance around $10 \Omega$ due to the two-lead contact geometry with large areas of the thin metal layer. We interpret our data in terms of a non-fluctuating offset resistance. For short samples we obtain rms amplitudes of $\Delta G \approx 0.24$ $e^{2} / h$. With our experimental parameters the length $L_{T}=\left(h D / e V_{m}\right)^{1 / 2}$ is of the same order of magnitude or smaller than the phase coherence length $l_{\varphi}$ and therefore influences the interference paths contributing to the fluctua- tion patterns. We have investigated the dependence of the typical "period" $B_{c}$ of the fluctuations on the width of the samples. For short samples with $L \approx L_{T}$ we find that $B_{c}$ is inversely proportional to the width in agreement with the picture that all electron paths within the phase coherence area contribute to the fluctuation pattern. For a very long sample with $L \gg L_{T}, l_{4}$ we also observe reproducible fluctuations with very small amplitude.

\section{ACKNOWLEDGMENTS}

The authors would like to thank $\mathrm{H}$. Heibrock for performing the e-beam writing. and P. Pfundstein for the electron microscopy. We also thank A. Sypli for her support in sample preparation and G. Goll for his help in the low temperature measurements. We are very indebted to R. Schäfer, K. Hecker, and H. Hegger for helpful discussions. This work was performed within the research program of Sonderforschungsbereich 195 supported by the Deutsche Forschungsgemeinschaft.

'S. Washburn and R. A. Webb. Adv. Phys. 35, 3375 (1986).

${ }^{2}$ P. A. Lee and A. D. Stone, Phys. Rev. Lett. 55, 1622 (1985).

${ }^{3}$ V. Chandrasekhar, D. E. Prober, and P. Santhanam, Phys. Rev. Lett. 61. 2253 (1988).

${ }^{4}$ W. Langheinrich, H. Beneking. U. Murek, C. Braden. and D. Wohlleben, J. Vac. Sci, Technol. B 9, 2904 (1991); U. Murek. R. Schäfer. and W. Langheinrich, Phys. Rev. Lett. 70. 841 (1993).

${ }^{5}$ M. Büttiker, Phys. Rev. Lett. 57, 1761 (1986).

${ }^{6}$ A. D. Benoit. C. P. Umbach, R, B. Laibowity, and R. A. Webb, Phys. Rev. Lett. 58, 2343 (1987); H. Haucke, S. Washburn, A. D. Benoit, C. P. Umbach, and R. A. Webb. Phys. Rev. B 41. $12454(1990)$

C. P. Umbach, S. Washburn, R. B. Laibowitz, and R. A. Webb. Phys. Rev. B 30, 4048 (1984) 